\title{
CW Performance of an InGaAs-GaAs-AlGaAs Laterally-Coupled Distributed Feedback (LC-DFB) Ridge Laser Diode
}

R. D. Martin, Student Member, IEEE, S. Forouhar, S. Keo, R. J. Lang, Member, IEEE,

R. G. Hunsperger, Fellow, IEEE, R. C. Tiberio, Member, IEEE, and P. F. Chapman

\begin{abstract}
Single-mode distributed feedback (DFB) laser diodes typically require a two-step epitaxial growth or use of a corrugated substrate. We demonstrate InGaAs-GaAs-AIGaAs DFB lasers fabricated from a single epitaxial growth using lateral evanescent coupling of the optical field to a surface grating etched along the sides of the ridge. $A \mathrm{CW}$ threshold current of $25 \mathrm{~mA}$ and external quantum efficiency of $0.48 \mathrm{~mW} / \mathrm{mA}$ per facet were measured for a $1 \mathrm{~mm}$ cavity length device with anti-reflection coated facets. Single-mode output powers as high as $11 \mathrm{~mW}$ per facet at $935 \mathrm{~nm}$ wavelength were attained. A coupling coefficient of at least $5.8 \mathrm{~cm}^{-1}$ was calculated from the subthreshold spectrum taking into account the $2 \%$ residual facet reflectivity.
\end{abstract}

\section{INTRODUCTION}

$\mathbf{S}$ TABLE single longitudinal mode behavior, narrow linewidths $(\sim \mathrm{MHz})$, low power requirements, and small size have made distributed feedback (DFB) semiconductor laser diodes attractive in many applications including spectroscopy, pump sources for amplifiers, injection sources for solid-state lasers, and as both sources and local oscillators in coherent communication systems. DFB laser diodes achieve wavelength selectivity by incorporating a periodic change of refractive index or gain along the lasing cavity. This is usually done by growing the laser structure on a corrugated substrate or by interrupting the growth above the active region, patterning and etching the grating, and then growing the upper cladding and cap layers. Determining the proper surface preparation and growth parameters to achieve high quality epitaxial regrowth while preserving the grating structure is technically demanding--particularly for short

Manuscript received September 20, 1994. This work was performed in part at the Center for Space Microelectronics Technology, Jet Propulsion Laboratory, California Institute of Technology, sponsored by the Ballistic Missile Defense Organization. Innovative Science and Technology Office and also at the National Nanofabrication Facility, which is supported by the National Science Foundation under Grant ECS-8619049, Comell University, and industrial affiliates. R. Martin was supported by a NASA Graduate Student Research Fellowship.

R. D. Martin and R. G. Hunsperger are with the Department of Electrical Engineering, Universily of Delaware, Newark, DE 19716 USA.

S. Forohuhar and S. Keo are with the Center for Space Microelectronics Technology, Jet Propulsion Laboratory, California Institute of Technology, Pasadena, CA 91109 USA.

R. J. Lang was with the Center for Space Microelectronics Technology, Jet Propulsion Laboratory. He is now with the SDL, Inc., San Jose, CA 95134.

R. Tiberio and $P$. Chapman are with the National Nanofabrication Facility. Cornell University, Ithaca, NY 148.53 USA

IEEE Log Number 9408433

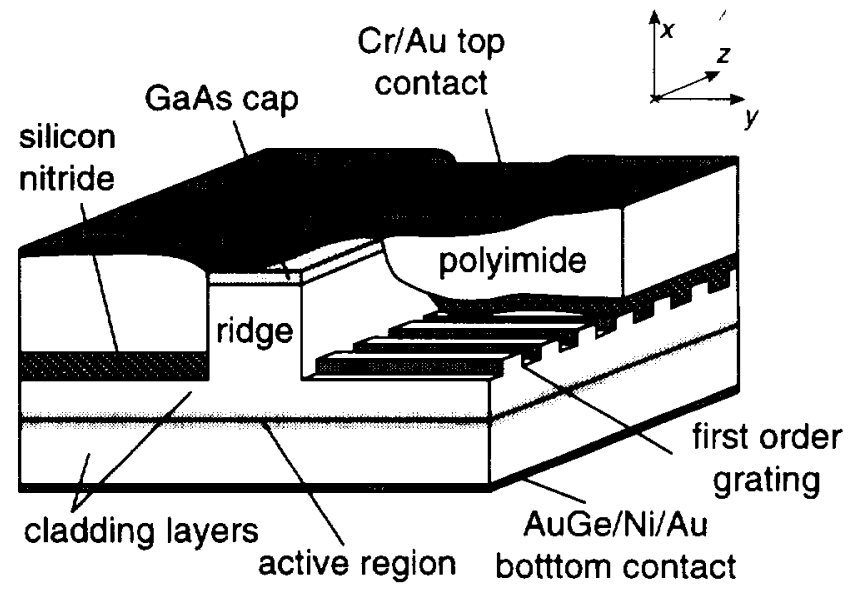

Fig. 1. Diagram of a laterally-coupled distributed feedback (LC-DFB) ridge laser diode.

wavelength $\mathrm{AlGaAs}$ lasers with high aluminum content and long wavelength GaSb-based devices.

One way to eliminate the regrowth problem is to rely on evanescent coupling of the electromagnetic fields to a surface grating. This approach has been demonstrated by etching the grating directly over the waveguide and injecting the current from the side [1] or by etching the grating through the cap and upper cladding layer to provide the lateral index guiding for the "ridge" and selective feedback [2]. Both of these techniques require a deep, uniform transfer of the grating into the upper cladding. An alternative approach is to etch the ridge first and then define the grating on either side of the ridge (Fig. 1) by either electron beam [3]-[4] or x-ray [5] lithography. The difficulty with this structure lies in achieving sufficient interaction of the evanescent field with the surface grating. Agrawal and Dutta have shown that the threshold of a ridge DFB increases by a factor of 20 or more when the gratings under the ridge are removed [6]. In this letter the $\mathrm{CW}$ lasing characteristics of a laterally-coupled distributed feedback (LCDFB) ridge laser using a single growth step are presented.

\section{FABRICATION}

The fabrication of LC-DFB lasers is very similar to that of standard ridge lasers. Photolithography was used to define $2 \mu \mathrm{m}$-wide photoresist stripes on an MBE grown InGaAs-GaAs-AlGaAs graded index separate confinement heterostructure (GRINSCH). Chemically assisted ion beam etch- 
ing (CAIBE) using chlorine gas in conjunction with an argon ion beam was used to etch the ridges to within $0.1 \mu \mathrm{m}$ of the GRINSCH. The two dimensional overlap of the electric field with the grating-and hence the coupling coefficient, $k$-is critically dependent on the ridge etch depth and width [7]. Etching the ridge too deep results in strong index guiding of the field and reduces the lateral ( $y$-direction) fill factor of the grating. Too shallow of a ridge depth reduces the transverse ( $x$-direction) overlap of the field with the grating. A narrow ridge was used to increase the lateral overlap of the electric field with the grating.

After etching the ridge, PMMA was applied to the wafer and a first order grating was exposed (Fig. 2) using electron beam lithography. To simplify alignment of the - -beam pattern to the ridge, the grating was written continuously across the structure. Grating free ridges were included in the pattern to permit comparison of Fabry-Perot and DFB devices from the same wafer. CAIBE was then used to transfer the grating approximately $700 \AA$ deep into the cap layer and upper cladding beside the ridge. The grating depth was much less than the thickness of the highly-doped cap region. The high resolution e-beam configuration used to expose the grating pattern resulted in very narrow exposed lines $(\sim 25 \mathrm{~nm})$ yielding an etched to unetched aspect ratio of $1: 5(16.6 \%$ duty cycle) for the etched grating. For a rectangular shaped first order grating, optimal feedback is achieved with a symmetric grating-i.e., a 50\% duty cycle. A self-aligned technique using silicon nitride and polyimide was used to planarize the wafer and facilitate contacting the narrow ridge. The wafer was thinned and finished with $\mathrm{Cr}-\mathrm{Au}$ ( $p$-type) and $\mathrm{AuGe}-\mathrm{Ni}-\mathrm{Au}$ (n-type) contacts. Devices were mounted junction side up on copper heat sinks for testing. Anti-reflection coatings consisting of a quarter wavelength $(\lambda / 4)$ of $\mathrm{Al}_{2} \mathrm{O}_{3}(\mathrm{R} \sim 2 \%)$ were deposited on both facets of the DFB devices to suppress Fabry-Perot modes.

\section{MEASUREMENTS AND ANALYSIS}

Laterally-coupled DFB lasers with cavity lengths of 1.0 and $1.5 \mathrm{~mm}$ had as-cleaved $\mathrm{CW}$ threshold currents of $10-15 \mathrm{~mA}$ which increased to 18-25 $\mathrm{mA}$ when AR coated. Power-current (P-I) characteristics of a $1.0 \mathrm{~mm}$ cavity length LC-DFB laser with AR coatings are shown in Fig. 3. The threshold current and external slope efficiency are $25 \mathrm{~mA}$ and $0.48 \mathrm{~mW} / \mathrm{mA}$ per facet. LC-DFB devices with cavity lengths of $250 \mu \mathrm{m}$ to $500 \mu \mathrm{m}$ had CW threshold currents of $8-10 \mathrm{~mA}$ with ascleaved facets. When AR coated, however, threshold currents increased to $35-80 \mathrm{~mA}$ indicating insufficient grating feedback (i.e. weak coupling) for these cavity lengths.

The inset of Fig. 3 shows the $\mathrm{CW}$ spectral characteristics of a $1 \mathrm{~mm}$ device measured with a $3 / 4 \mathrm{~m}$ spectrometer as the current is increased from $30-45 \mathrm{~mA}$ in $5 \mathrm{~mA}$ steps. Singlemode DFB operation was observed up to $11 \mathrm{~mW}$ at which point a second DFB mode begins to lase. The side-mode suppression ratio (SMSR) measured with an optical spectrum analyzer was greater than $30 \mathrm{~dB}$ at an output power of $10 \mathrm{~mW}$. The lasing wavelength as a function of heat sink temperature was measured for a LC-DFB laser along with a standard ridge laser. The lasing wavelength of the ridge devices without

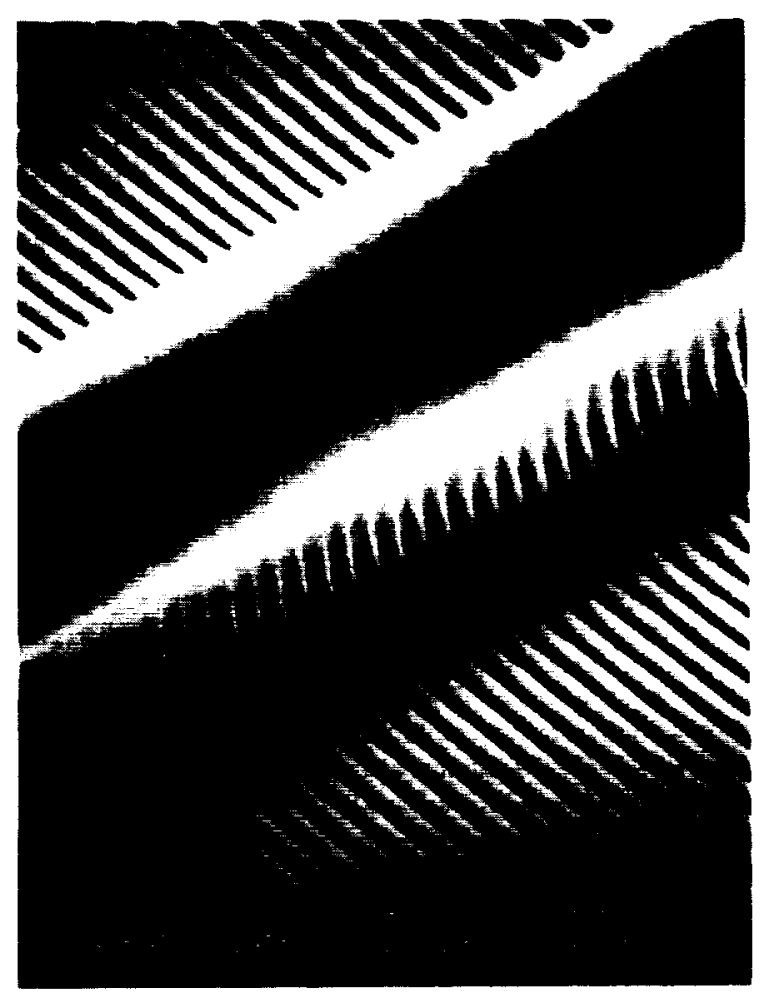

Fig. 2. Scanning electron micrograph of the first order grating pattern over the ridge defined in PMMA by '-beam lithography.

gratings shifted at $\sim 3 \AA /{ }^{\circ} \mathrm{C}$ compared to a much slower rate of $0.65 \AA /{ }^{\circ} \mathrm{C}$ for the LC-DFB devices.

The coupling coefficient can be determined from the subthreshold normalized stop-band width [8], WL, defined as the normalized mode spacing between the fundamental (lowest threshold) DFB mode and the lowest threshold adjacent DFB mode, i.e..

$$
W L=2 \pi n_{\cdot \mathrm{ff}} L\left|\frac{1}{\lambda}-\frac{1}{\lambda_{ \pm}}\right|=2 \pi n_{\cdot \mathrm{ff}} L \frac{\Delta \lambda}{\lambda^{2}} .
$$

In this equation $\lambda, \lambda_{-}$, and $\lambda_{+}$are the wavelengths of the fundamental, shorter adjacent, and longer adjacent modes, $n_{r+f f}$ is the effective index including the dispersion $|9|$, and $L$ is the laser cavity length. The relationship between $W L$ and $r L$ is shown in Fig. 4 for the case of $0 \%$ and $2 \%$ facet reflectivity. The perfect AR casc is calculated using the theory of Kogelnik and Shank [10]. It becomes more difficult to determine the coupling when the residual facet reflectivity is taken into account because the phase of the grating at the front and rear facets is unknown. For the $2 \%$ case, the phases of the grating at the front and rear facets were each varied from 0 to $15 \pi / 8$ in $\pi / 8$ steps. The normalized threshold gain $(\alpha L)$ and frequency deviation from the Bragg condition ( $\delta L$ ) of the 5 modes nearest the Bragg wavelength were calculated $[11]$ for each of the 256 phase combinations for $\kappa L$ values ranging from 0.2 to 1.0 . The minimum and maximum normalized stop-band width were extracted for each ri $L$ value. Fig. 4 shows that for a given value of $k L$, the residual reflectivity and random grating phase of the facets introduces a large variation in the stop-band width. WL values of $4.14(L=1.0 \mathrm{~mm})$ and $4.61(L=1.5 \mathrm{~mm})$ were measured on subthreshold spectra of LC-DFB lasers. Fig. 


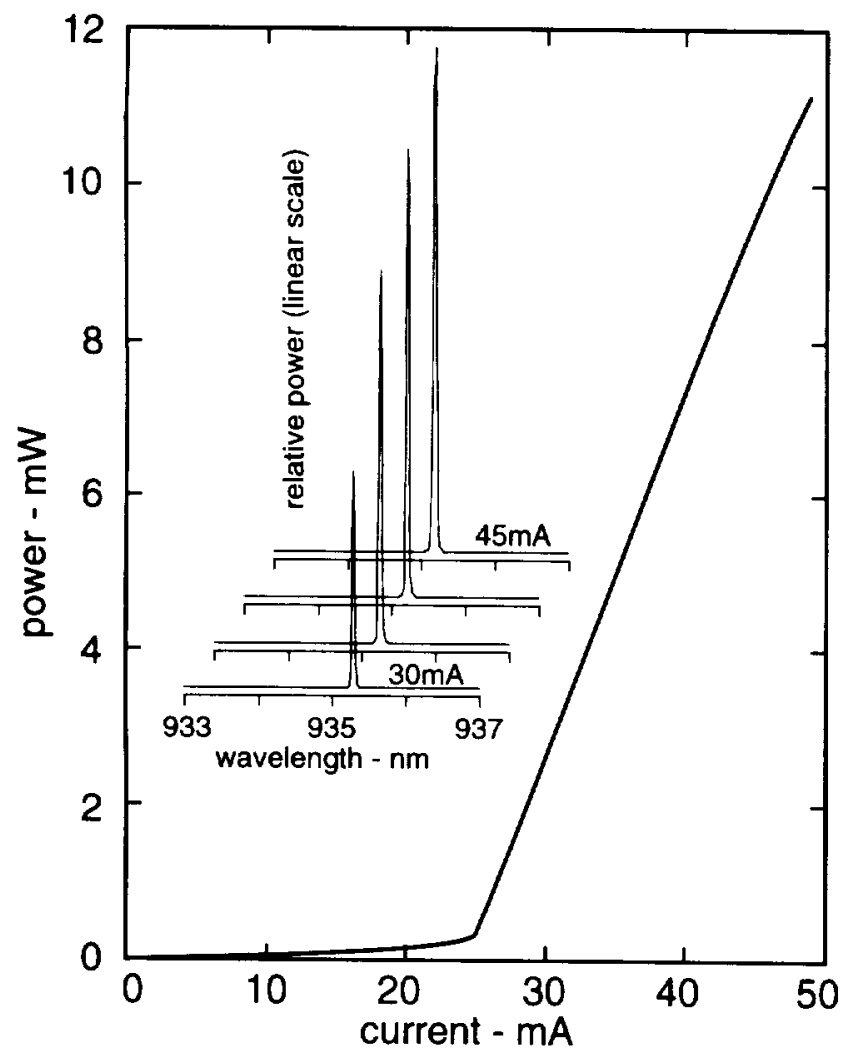

Fig. 3. CW power-current and spectral characteristics of a $1 \mathrm{~mm}$ cavity LC-DFB laser with AR-coated facets. For the spectral data the current is stepped from $30 \mathrm{~mA}$ to $45 \mathrm{~mA}$ in $5 \mathrm{~mA}$ steps.

4 shows that for the measured WL values, $\kappa L$ is at least 0.59 $\left(\kappa=5.9 \mathrm{~cm}^{-1}\right)$ and $0.87\left(\kappa=5.8 \mathrm{~cm}^{-1}\right)$ for the $1 \mathrm{~mm}$ and $1.5 \mathrm{~mm}$ devices respectively. In general, for good performance of a DFB laser, a $\kappa L$ product of $1-1.5$ is desired. A theoretical coupling coefficient of $8.6 \mathrm{~cm}^{-1}$ was determined by applying coupled-mode theory [7] to the InGaAs LC-DFB laser with a $16.6 \%$ duty cycle rectangular grating. This analysis revealed that the coupling coefficient would be doubled with a $50 \%$ duty cycle grating. If the minimum measured coupling value $\left(\kappa=5.8 \mathrm{~cm}^{-1}\right)$ is doubled by using a symmetric grating, a $\kappa L$ of 1.0 could be attained for a LC-DFB laser with a $900 \mu \mathrm{m}$ cavity length.

\section{SUMmaRY AND CONCluSION}

Single mode distributed feedback laser diodes were produced from a single epitaxial growth that rely on the lateral coupling of the evanescent electromagnetic fields with a surface grating etched along the sides of the ridge. Good performance of strained layer InGaAs-GaAs-AIGaAs SQW LC-DFB lasers was achieved. A $25 \mathrm{~mA} \mathrm{CW}$ threshold current, total external quantum efficiency of $82 \%$, and single longitudinal mode output power of $11 \mathrm{~mW}$ were attained for a $1 \mathrm{~mm}$ cavity length laterally-coupled device. The $2 \%$ residual facet reflectivity of the anti-reflection coating used on the LC-DFB lasers prevented an exact measurement of $r$ from the stop-band but taking into account the random phase of the grating at the facets, a minimum coupling coefficient of $5.8 \mathrm{~cm}^{-1}$ was determined from the subthreshold spectra. We have demonstrated that a laterally-coupled DFB laser has

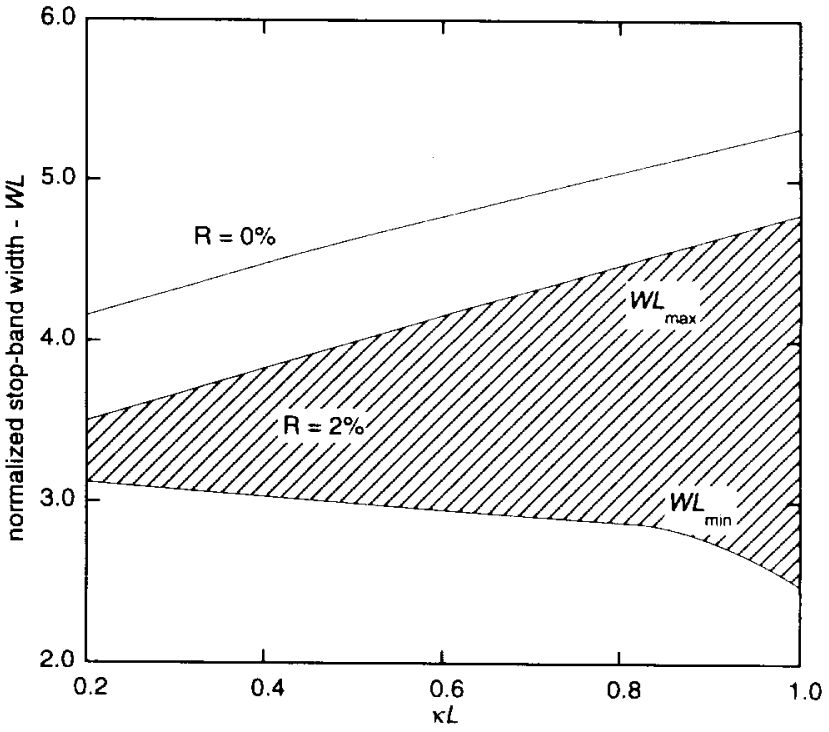

Fig. 4. Relationship between the normalized stop-band width, WL, and $\kappa L$ for residual facet reflectivity of $0 \%$ and $2 \%$. For the $2 \%$ case, the phases of the grating at each facet were varied from 0 to $15 \pi / 8$ in $\pi / \delta$ steps. The minimum and maximum normalized stop-band width obtained from the 256 phase combinations are shown.

potential as a stable, single-mode device with power levels suitable for many applications. This technique could be used to fabricate DFB lasers in material systems for which regrowth is prohibitive - such as high $\mathrm{Al}$ concentration (short wavelength and visible lasers) and GaSb (long wavelength) based devices.

\section{REFERENCES}

11 Z. L. Liau, D. C. Flanders, J. N. Walpole, and N. L. DeMeo, "A novel GaInAsP/nnP distributed feedback laser," Appl. Phys. Lett., vol, 46. no. 3, pp. $221-223,1$ Feb. 1985.

[2] L. M. Miller, K. J. Beernink, J. T. Verdeyen, J. J. Coleman. J. S. Hughes, G. M. Smith, J. Honig, and T. M. Cockerill, "Characterization of an InGaAs-GaAs-AlGaAs strained-layer distributed-feedback ridgewaveguide quantum-well helerostructure laser," IEEE Photon. Terthol. Lett. vol. 4, no. 4. pp. 296-299, Apr. 1992.

[3] R. D. Martin, S. F. Forouhar, S. Keo, R. J. Lang. R. G. Hunsperger, R. C. Tiberio, and P. F. Chapman. "InGaAs-GaAs-AlGaAs laterally-coupled distributed feedback (LC-DFB) ridge laser diode," Elertron. Letr., vol. 30, no. 13, pp. 1058-1060, 23 Jun. 1994.

[4] R. C. Tiberio, P. F. Chapman, R. D. Martin, S. F. Forouhar, and R. J. Lang, "Laterally coupled distributed feedback lasers fabricated with electron beam lithography and chemically assisted ion beam etching." to be published in the J. of Vac. Scie. and Technol. B.

$[5]$ V. V. Wong, W. Choi, J. Carer, C. G. Fonstad, and H. I. Smith. "Ridgegrating distributed-feedback lasers fabricated by $x$-ray lithography." $I$. of Vac. Scie and Techmol. B. vol. 11, p. 2621, 1993.

[6] G. P. Agrawal and N. K. Dutta, "Analysis of ridge-waveguide distributed feedback lasers," IEEE J. of Quantum Electron., vol. QE-21, no. 6, pp. 534-538, Jun, 1985.

[7] R. D. Martin, S. Forouhar, R. J. Lang. and R. G. Hunsperger, unpublished.

[8] K. Kihara. H. Soda. H. Ishikawa, and H. Imai. "Evaluation of the coupling coefficient of a distributed feedback laser with residual facet reflectivity." J. of Appl. Phys., vol. 62, no. 4, pp. 1526-1527. 15 Aug. 1987.

[9] K. Iga, "On the use of the effective refractive index in DFB laser mode separation," Japan. J. of Appl. Phys, vol. 22, no. 10, p. 16.30. 1983.

$|10| \mathrm{H}$. Kogelnik and C. V. Shank. "Coupled-wave theory of distributed feedback lasers," J. of Appl. Phys, vol. 43, no. 5. pp. 2327-23.35, May 1972.

H11] W. Streifer. R. D. Burnham, and D. R. Scifres, "Effect of external refiectors on tongitudinal modes of distributed feedback lasers," IEEE J. of Quantum Ele'tron., vol. QE-11, no. 4. pp. 154-161, Apr. 1975. 
